\title{
Ocular Sparganosis: The First Report of Spirometra ranarum in Thailand
}

\author{
Wilai Saksirisampant ${ }^{1, \dagger}{ }^{1}$, Chatanun Eamudomkarn ${ }^{2,3, \dagger}$, Hyeong-Kyu Jeon ${ }^{2}$ [D, Keeseon S. Eom², \\ Buravej Assavapongpaiboon ${ }^{4}$, Sunisa Sintuwong ${ }^{5}$, Wasee Tulvatana, ${ }^{4, *}$ (i) \\ 'Department of Parasitology, Faculty of Medicine, Chulalongkorn University, Bangkok 10330, Thailand; ²Department of Parasitology, Parasite Research \\ Center and Parasite Resource Bank, Chungbuk National University School of Medicine, Cheongju 28644, Korea; ${ }^{3}$ Department of Parasitology, Faculty \\ of Medicine, Khon Kaen University, Khon Kaen 40002, Thailand; ${ }^{4}$ Department of Ophthalmology, Faculty of Medicine, Chulalongkorn University, \\ Bangkok 10330, Thailand; 5 Mettapracharak Eye Centre, Mettapracharak (Wat Raikhing) Hospital, Nakhon Pathom 73210, Thailand
}

\begin{abstract}
A 22-year-old Thai man from the Northeast region presented with acute eye swelling, itching, and discharge on his left eye. He was suspected of having gnathostomiasis and treated with albendazole and prednisolone for 3 weeks. Nine months later, he was treated with high-dose oral prednisolone for the preliminary and differential diagnoses with thyroid-associated orbitopathy and lymphoma. He had been administered prednisolone intermittently over a few years. Then he developed a painless movable mass at the left upper eyelid and recurrent pseudotumor oculi was suspected. The surgical removal of the mass was performed. A white pseudosegmented worm revealed a definite diagnosis of ocular sparganosis by a plerocercoid larva. Molecular diagnosis of the causative species was made based on the mitochondrial cytochrome c oxidase subunit 1 (cox1) gene. Proper technique of extraction and amplification of short fragments DNA from formalin-fixed paraffin-embedded tissue successfully identified parasite species. The result from the sequencing of the PCR-amplified cox1 fragments in this study showed $99.0 \%$ sequence homology to Spirometra ranarum. This is the first report of $S$. ranarum in Thailand.
\end{abstract}

Key words: Ocular sparganosis, Spirometra ranarum, Thailand

\section{INTRODUCTION}

Sparganosis is a zoonotic disease caused by infection with the plerocercoid larvae or sparganum stages of Spirometra species (diphyllobothridean =pseudophyllidean) tapeworm. Adult worms reside in the intestines of the definitive host (dogs and cats). Human infection occurs accidentally by ingesting contaminated water with copepods or undercooked meat of amphibian, fish, and bird or using infected meat of snakes as a poultice [1]. After passing through the intestinal wall, spargana then migrate through the body tissues. The larva can invade the subcutaneous tissue, the central nervous system, breast, and eyes [1]. The common site of the infection is subcutaneous tissue which is about half of the reported cases in Thailand $[1,2]$. The ocular site is rare which is present in the eyelid and

- Received 3 April 2020, revised 19 August 2020, accepted 22 August 2020.

*Corresponding author (waseetulvatana@gmail.com)

†These authors contributed equally to this work.

(c) 2020, Korean Society for Parasitology and Tropical Medicine

This is an Open Access article distributed under the terms of the Creative Commons Attribution Non-Commercial License (https://creativecommons.org/licenses/by-nc/4.0) which permits unrestricted non-commercial use, distribution, and reproduction in any

medium, provided the original work is properly cited. subconjunctival swelling with pain, itching, and epiphora. These should be differentiated from allergic blepharitis, conjunctivitis, and other common tissue nematodes of gnathostomiasis $[3,4]$. The larva morphology is species undistinguished. By molecular characterization, various species were recognized by many investigators worldwide. S. mansoni is responsible for this disease in Asia, whereas $S$. mansonoides is important in North America [5,6]. S. erinaceieuropaei, S. decipiens, S. ranarum, and $S$. theileri were more recently reported as valid from the United States, Myanmar, Korea, China, Japan, and Tanzania [7-10]. In Thailand, S. erinaceieuropaei was the only species reported [11]. In this study, we reported an ocular sparganosis from a Thai patient that was identified, based on the morphological and histological examinations on the plerocercoid larva. The Spirometra species was identified by molecular analysis of cytochrome $c$ oxidase 1 ( $\operatorname{cox} 1)$.

\section{CASE DESCRIPTION}

The ethics review was exempted by the Institutional Review Board of the Faculty of Medicine, Chulalongkorn University, 
Bangkok, Thailand (COE No. 003/2020, IRB No. 844/62).

\section{History}

A 22-year-old Thai man from the Northeast region came to a hospital in Nakhon Pathom province of Thailand with an acute eyelid swelling, itching, and conjunctival discharge on his left eye. Physical examination first revealed swelling and ecchymosis both upper and lower lids and subconjunctival hemorrhage. With a history of consuming raw home-made pickled freshwater fish, gnathostomiasis was suspected.

\section{Laboratory findings and treatment}

He was empirically treated for gnathostomiasis with albendazole and prednisolone for 3 weeks. The eyelid swelling decreased but reappeared after these medications were stopped. Throughout clinic visits, visual acuity (VA), fundus examination and eye tension were normal.

Nine months later, chronic binocular diplopia with itching, pain, and swelling on the left eye of the patient were present. Blood tests such as $\mathrm{CBC}$ and absolute eosinophil count were within normal limits. Gnathostoma antibody showed positive IgG ELISA and a weakly positive IgG Western blot to $24 \mathrm{kDa}$. The physical examination showed vertical diplopia and normal VA and tension. CT orbit demonstrated left proptosis with unilateral diffuse enlargement of extraocular muscles, especially at superior rectus, superior oblique and medial rectus muscles with mild retro-orbital and orbital apex fatty infiltration. The sclera was mildly enhanced and slightly thickened. An idiopathic orbital inflammatory disease (pseudotumor oculi) was preliminarily diagnosed and differential diagnoses included thyroid-associated orbitopathy and lymphoma. ESR, thyroid hormone, VDRL, rheumatoid factor, and anti-HIV were then tested and showed negative results. High-dose oral prednisolone $(1 \mathrm{mg} / \mathrm{kg} /$ day) was given for 3 weeks with the tapering course. The lid swelling was initially decreased after treatment. However, he still suffered multiple episodes of eyelid swelling and proptosis. He had been treated with prednisolone intermittently over a few years. Then he developed a painless movable mass at the left upper eyelid and recurrent pseudotumor oculi were suspected.

\section{Surgery}

On the movable mass, surgical removal was done. A white plerocercoid larva was revealed (Fig. 1A) and sent to the ophthalmology and parasitology departments of the King Chulalongkorn Memorial Hospital, Bangkok. The pathological section (Fig. 1B) of the parasite tissue showed a single layer of thick tegument (arrow), loose parenchyma cells, laminated basophilic calcareous corpuscles (calcium carbonate), and a presence of longitudinal muscle bundles without body cavity. Ocular sparganosis was diagnosed. Prednisolone with a tapering dose was given. The proptosis and swelling resolved and have not recurred.

\section{Molecular identification of the specimen}

To extract DNA for molecular identification of the Spirometra species, $10 \mu \mathrm{m}$-thicknesses of unstained thin sections (cut from the formalin-fixed paraffin-embedded (FFPE) specimen) were used. The sections were deparaffinized by dissolved in xylene, followed by absolute ethanol. Genomic DNA was extracted
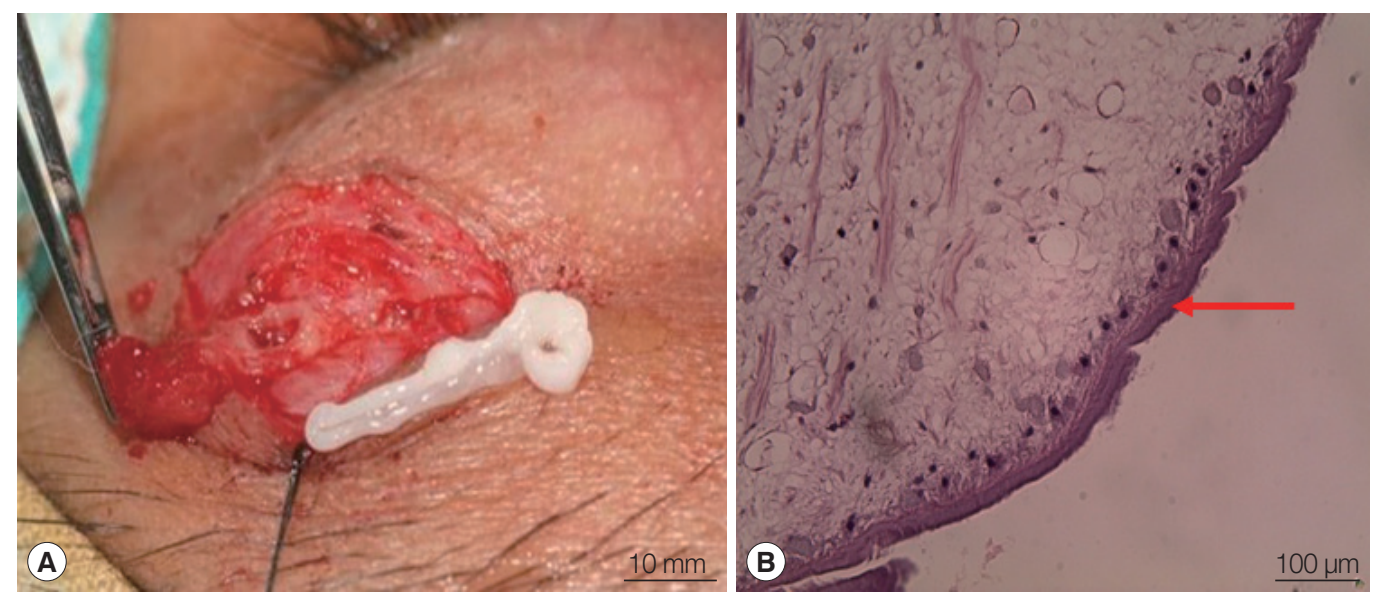

Fig. 1. (A) Whitish plerocercoid larva was revealed from the upper eyelid during surgical removal of the mass. (B) Histological finding (hematoxylin and eosin stain). 
from QIAamp ${ }^{\circledR}$ DNA FFPE Tissue Kits (QIAGEN, Hilden, Germany) according to the manufacturer's instructions. The DNA was eluted in $30 \mu \mathrm{l}$ of sterilized distilled water and used as a template for whole genome amplification. DNA was amplified by GenomePlex ${ }^{\circledR}$ Complete Whole Genome Amplification (WGA) Kit (Sigma Aldrich, St. Louis, Missouri, USA) according to the manufacturer's instructions.

The mitochondrial cytochrome $\mathrm{c}$ oxidase subunit 1 (cox 1$)$ gene was targeted in PCR amplification. The partial fragments of the cox 1 gene were amplified by PCR. PCR reactions were performed in a $20 \mu \mathrm{l}$ reaction volume consisting of $4 \mu \mathrm{l}$ of HiPi Plus $5 \times$ PCR master mix (Elpis Biotech. Inc., Daejeon, Korea), 10 pmol of each primer, $2 \mu \mathrm{l}$ of extracted DNA, and nuclease-free water added up to a final volume. Reactions were performed in a thermocycler (Biometra Co., Goettingen, Germany). Thermocycling conditions included an initial denaturation at $94^{\circ} \mathrm{C}$ for $3 \mathrm{~min}, 35$ cycles of $94^{\circ} \mathrm{C}$ for $1 \mathrm{~min}$, annealing at $46^{\circ} \mathrm{C}$ for $40 \mathrm{sec}$, and then extension at $72^{\circ} \mathrm{C}$ for $1 \mathrm{~min}$, followed by a final extension at $72^{\circ} \mathrm{C}$ for $10 \mathrm{~min}$. Each set of experiments included negative and positive controls. Nucleasefree water replaced DNA templates for negative controls.

The PCR primers were designed based on cox 1 sequences of S. erinaceieuropaei and S. decipiens. The primer was used to amplify the cox 1 region as follows; pcox4f: 5'-CAT GGT TGC TTT TTC CTT-3' and pcox4r: 5'-ACT ACG AGA CAC AAA AYT ATC 3', pcox6f: 5'-CTA TTC CTG TGT TGG CTG CT-3' and pcox6r: 5'-TAT CAT AWG AAC AAC CTA ATT TAC-3', pcoxOf: 5'-GGK ATA AAG GTG TTT TCT TG-3' and pcox9r: 5'-ATM ACA TAA TGA AAA TGA GCC ACC-3', pcox10f: 5'-GGK GGT GTT ACY GGT ATA AT-3' and pcox10r: 5'-GAY ACT ATA CAA TGA CAC TGT-3'.

PCR products were confirmed by $1 \%$ agarose gel electrophoresis and purified using DokDo-Prep PCR Purification Kit (Elpis Biotech) for direct DNA sequencing. Sequence analysis was performed using Geneious Program (Biomatters Co., Auckland, New Zealand).
For the molecular analysis of the plerocercoid, 4 of $\operatorname{cox} 1$ fragments were amplified successfully. The fragments were corresponded to position 360-483 (123 bp), 643-800 (157 bp), 986-1,097 (111 bp) and 1,102-1,254 (152 bp) of cox1 gene. Homology search of the PCR-amplified products was conducted against regions of the mitochondrion, the complete genome of S. erinaceieuropaei (GenBank No. KJ599680.1), S. theileri (GenBank No. MK955901.1), S. ranarum (GenBank No. MN264212.1) and S. decipiens (GenBank No. KJ599679.1) have been presented. Our results were summarized and shown in Table 1. All of 4 fragments sequences showed high similarities of $S$. ranarum. The total length of the sequence was $543 \mathrm{bp}$ showed 99\% sequence homology to S. ranarum. The sequences of $S$. ranarum were submitted to the GenBank database under numbers GenBank No. MT590763.1. Therefore, the plerocercoid from the ocular sparganosis patient was identified as $S$. ranarum.

\section{DISCUSSION}

This study is the first report of $S$. ranarum from a sparganosis patient in Thailand. We showed that proper technique of extraction and amplification of short fragments DNA from FFPE tissue allowed us to speculate successfully of the parasite. The molecular technique is a powerful tool for species identification. S. ranarum was reported first time by Gastaldi (1854) in Italy as Diphyllobothrium ranarum found in the frog (Rana tigrina) $[8,12]$. By molecular analysis based on mitochondrial genome analysis, the 2 most common species of $S$. erinaceieuropaei and S. decipiens have been reported in Asian [11,13-15]. Recently, S. ranarum was reported from frogs in Myanmar by using mitochondrial cox 1 and nad1 genes [8]. The species identification from the study was based on morphology and molecular analysis. The authors found that sequence differences in cox 1 and nad 1 genes were $2.1 \%$ between $S$. ranarum and S. decipiens and were 9.5\% (cox1) and 11.9\% (nad1) between

Table 1. Sequence homology of the PCR-amplified cox1 fragments

\begin{tabular}{lcccc}
\hline \multirow{2}{*}{ FFPE sample position (bp) } & \multicolumn{4}{c}{ cox1 sequence homology against } \\
\cline { 2 - 5 } & S. ranarum (\%) & S. decipiens (\%) & S. erinaceieuropaei (\%) & S. theileri (\%) \\
\hline $360-483$ & 98.4 & 94.3 & 91.1 & 87.8 \\
$643-800$ & 99.4 & 97.5 & 91.8 & 90.5 \\
$986-1,097$ & 98.2 & 97.3 & 91.0 & 86.5 \\
$1,102-1,254$ & 100.0 & 98.7 & 91.5 & 86.9 \\
Average & 99.0 & 97.0 & 91.4 & 87.9 \\
\hline
\end{tabular}


S. ranarum and S. erinaceieuroapei [8]. The patient was from the Northeast region of the country where maybe possible intercontinental or intracontinental traveling or the autochthonous infection as well.

The first case report of sparganosis in Thailand was in 1943, and until 2002, there were 34 reported cases in which half $(50.0 \%)$ of these were on the ocular site [16]. Another literature review showed $1 / 3$ of sparganosis patients had ocular infection [2]. Molecular studies of Spirometra in Thailand have a limit. The parasites from a recent report in 9 cases from a tertiary hospital, S. erinaceieuropaei were identified as based on cox1 gene [11].

The ocular site of the disease reported is challenging. The diagnosis can initially be missed as gnathostomiasis, which is highly prevalent and have a similar clinical presentation [4]. Gnathostomiasis is unlikely in this case because there was no improvement after the 3-week treatment of albendazole. Moreover, there was no clue of the absolute eosinophil count. Due to the common antigenic epitope among helminth could have ELISA been strongly positive and a more specific technique of Western blot showed a weak reaction in this case [17]. Nevertheless, past infection of gnathostomiasis might not be excluded.

Ocular sparganosis usually presents with eyelid and subconjunctival swelling which should be differentiated from allergic blepharitis and conjunctivitis. The common signs and symptoms include complaints of lacrimation, itching, pain, eye redness, and swelling of the eyelid. Other less common ocular presentation includes intraorbital mass in the soft tissue, endophthalmitis, and blindness [18,19]. Generally, patients have only one lesion, caused by a single sparganum larva. However, there was a case report with more than one lesion with the presence of spargana in each lesion [20].

It is also difficult to differentiate ocular sparganosis from myositic idiopathic orbital inflammatory disease (IOI) as well. As a radiologic finding, even with the recently proposed diagnostic criteria of myositic IOI, misdiagnosis occurs due to partial improvement of symptoms after corticosteroid therapy $[3,19]$. Most ocular sparganosis cases are diagnosed by the discovery of sparganum from infected tissue, mainly from the conjunctiva and eyelid, as seen with this case. Complete surgical removal is needed; otherwise, sparganosis can recur by regeneration of the larva that was left behind.

In this case, with a history of eating raw fish, the patient was probably infected with plerocercoid larvae of Spirometra spp. in fish. However, due to the prevalence of infection, gnathostomiasis, which can produce a similar clinical picture, was initially suspected [4]. Multiple courses of albendazole, as well as prednisolone, were given with intermittent improvement of symptoms. The steroid treatment allowed the orbital inflammation to partially subside, but the treatment did not kill the worm. In the later course, the patient developed symptoms of proptosis and ophthalmoplegia and, with the evidence on CT scan, IOI was diagnosed. Multiple repeated doses of prednisolone were given with partial improvement. Finally, the diagnosis was reached by the eyelid mass removing.

In conclusion, this is the first report of $S$. ranarum ocular sparganosis in Thailand. The ocular cases mostly presented with superficial ocular mass lesions. Before the diagnosis of orbital inflammation, this infection should be considered. The main treatment is the surgical removal of the parasite. Gross morphology with deep invagination and histology finding of the plerocercoid larva are key features for diagnosis. Molecular speciation was a promising technique for the parasite species. Public health surveillance programs are recommended for a person to avoid consuming untreated natural water and insufficiently cooked meat.

\section{ACKNOWLEDGMENTS}

We appreciated the kindly help from Ms. Jaruratt Prownebon and Dr. Atchara Pumee, the staff of the Department of Parasitology, Faculty of Medicine, Chulalongkorn University, Thailand for the specimen preparation.

\section{CONFLICT OF INTEREST}

The authors have no potential conflicts of interest to disclose.

\section{REFERENCES}

1. Liu Q, Li MW, Wang ZD, Zhao GH, Zhu XQ. Human sparganosis, a neglected food borne zoonosis. Lancet Infect Dis 2015; 15: 1226-1235.

2. Anantaphruti MT, Nawa Y, Vanvanitchai Y. Human sparganosis in Thailand: an overview. Acta Trop 2011; 118: 171-176.

3. Mombaerts I, Bilyk JR, Rose GE, McNab AA, Fay A, Dolman PJ, Allen RC, Devoto MH, Harris GJ. Consensus on diagnostic criteria of idiopathic orbital inflammation using a modified Delphi approach. JAMA Ophthalmol 2017; 135: 769-776. 
4. Bussaratid V, Dekumyoy P, Desakorn V, Jaroensuk N, Liebtawee B, Pakdee W, Wattanagoon Y. Predictive factors for Gnathostoma seropositivity in patients visiting the gnathostomiasis clinic at the hospital for tropical diseases, Thailand during 2000-2005. Southeast Asian J Trop Med Public Health 2010; 41: 1316-1321.

5. Mueller JF. The biology of Spirometra. J Parasitol 1974; 60: 3-14.

6. Richardson DJ. Other noteworthy zoonotic helminths. In Richardson DJ, Krause PJ eds, North American Parasitic Zoonoses. Springer Science \& Business Media. New York, USA. 2012, pp 87.

7. Jeon HK, Park H, Lee D, Choe S, Sohn WM, Eom KS. Molecular Detection of Spirometra decipiens in the United States. Korean J Parasitol 2016; 54: 503-507.

8. Jeon HK, Park H, Lee D, Choe S, Kang Y, Bia MM, Lee SH, Sohn WM, Hong SJ, Chai JY, Eom KS. Genetic and morphologic identification of Spirometra ranarum in Myanmar. Korean J Parasitol 2018; 56: 275-280.

9. Jeon HK, Eom KS. Mitochondrial DNA sequence variability of Spirometra species in Asian countries. Korean J Parasitol 2019; 57: 481-487.

10. Eom KS, Park H, Lee D, Choe S, Kang Y, Bia MM, Ndosi BA, Nath TC, Eamudomkarn C, Keyyu J, Fyumagwa R, Mduma S, Jeon HK. Identity of Spirometra theileri from a Leopard (Panthera pardus) and Spotted Hyena (Crocuta crocuta) in Tanzania. Korean J Parasitol 2019; 57: 639-645.

11. Boonyasiri A, Cheunsuchon P, Suputtamongkol Y, Yamasaki H, Sanpool O, Maleewong W, Intapan PM. Nine human sparganosis cases in Thailand with molecular identification of causative parasite species. Am J Trop Med Hyg 2014; 91: 389-393.

12. Faust EC, Campbell HE, Kellogg CR. Morphological and bio- logical studies on the species of Diphyllobothrium in China. Am J Epidemiol 1929; 9: 560-583.

13. Jeon HK, Park H, Lee D, Choe S, Kim KH, Huh S, Sohn WM, Chai JY, Eom KS. Human infections with Spirometra decipiens Plerocercoids identified by morphologic and genetic analyses in Korea. Korean J Parasitol 2015; 53: 299-305.

14. Okamoto M, Iseto C, Shibahara T, Sato MO, Wandra T, Craig PS, Ito A. Intraspecific variation of Spirometra erinaceieuropaei and phylogenetic relationship between Spirometra and Diphyllobothrium inferred from mitochondrial CO1 gene sequences. Parasitol Int 2007; 56: 235-238.

15. Jongthawin J, Intapan PM, Sanpool O, Sadaow L, Laymanivong S, Thanchomnang T, Maleewong W. Molecular evidence of Spirometra erinaceieuropaei infection in snakes Ptyas korros from Lao PDR and Thailand and frogs Hoplobatrachus rugulosus from Myanmar. Southeast Asian J Trop Med Public Health 2014; 45: 1271-1278.

16. Wiwanitkit V. A review of human sparganosis in Thailand. Int J Infect Dis 2005; 9: 312-316.

17. Nuchprayoon S, Sanprasert V, Suntravat M, Kraivichian K, Saksirisampant W, Nuchprayoon I. Study of specific IgG subclass antibodies for diagnosis of Gnathostoma spinigerum. Parasitol Res 2003; 91: 137-143.

18. Sen DK, Muller R, Gupta VP, Chilana JS. Cestode larva (Sparganum) in the anterior chamber of the eye. Trop Geogr Med 1989; 41: 270-273.

19. Ho TH, Lin MC, Yu WW, Lai PH, Sheu SJ, Bee YS. Ocular sparganosis mimicking an orbital idiopathic inflammatory syndrome. Orbit 2013; 32: 395-398.

20. Jipipop B, Chenpanich U. Ocular sparganosis with a case report. J Int Coll Surg Thai 1964; 7: 33-37. 
